\title{
ADMISSIBILITY, THE LOCALLY CONVEX APPROXIMATION PROPERTY, AND THE AR-PROPERTY IN LINEAR METRIC SPACES
}

\author{
NGUYEN TO NHU
}

(Communicated by James West)

\begin{abstract}
We introduce the notion of the locally convex approximation property (the LCAP) for convex sets in linear metric spaces. The LCAP is an extension of the notion of admissibility of Klee. We prove that any convex set with the LCAP is an AR.
\end{abstract}

\section{INTRODUCTION}

The following question (we call it the AR-problem) is among the most outstanding problems in infinite-dimensional topology:

1.1. Problem. Is every convex set in a linear metric space an AR? See [G, W, Problems LS1, LS6].

For locally convex spaces Problem 1.1 was settled affirmatively by Dugundji [D]. However, this problem remains open for nonlocally convex linear metric spaces and is one of the most resistant open problems in infinite-dimensional topology.

In this paper we provide some partial answers to the AR-problem. Our idea of attacking Problem 1.1 is to approximate convex sets in linear metric spaces by convex sets in locally convex spaces. We introduce the notion of the locally convex approximation property (the $L C A P$ ) for convex sets in linear metric spaces. The LCAP is an extension of the notion of admissibility of Klee [K1, K2] but has some advantages: In fact, we prove that the LCAP does imply the AR-property for all convex sets (not necessarily separable); meanwhile admissibility does not have this property: van der Bijl and van Mill [BM] have shown that even in the separable case admissibility does not imply the AR-property for convex sets unless all linear metric spaces are AR.

Roughly speaking, our theorem states that if a convex set $X$ can be "approximated", in some sense, by convex subsets in locally convex spaces, then $X$ is an AR. For instance, any convex set which is a union of an increasing sequence of convex subsets each of which can be affinely imbedded into a locally convex space, is an AR.

Received by the editors January 4, 1993 and, in revised form, March 10, 1994.

1991 Mathematics Subject Classification. Primary 54C55; Secondary 54D45.

Key words and phrases. Linear metric spaces, convex sets, AR-spaces, admissible convex sets, the locally convex approximation property.

The author was supported by the Spanish Ministry of Education.

(C) 1995 American Mathematical Society 
Our results in this paper provide new examples of convex sets with the ARproperty and raise some new problems for further investigation to the ARproblem in linear metric spaces, one of the most difficult problems in infinitedimensional topology.

Notation and Conventions. In this paper all maps are assumed to be continuous. By a linear metric space we mean a topological linear space $X$ which is metrizable. We assume that $X$ is equipped with an $F$-norm $\|\cdot\|$ such that $($ see $[\operatorname{Re}])$

$$
\|\lambda x\| \leq\|x\| \text { for every } x \in X \text { and } \lambda \in \mathbb{R} \text { with }|\lambda| \leq 1 .
$$

The zero element of $X$ is denoted by $\theta$. A locally convex space is a linear metric space which possesses a basis of neighbourhoods of $\theta$ consisting of convex sets.

Let $E$ be a subset of a linear space $X$. By conv $E$ we denote the convex hull of $E$ in $X$, and span $E$ denotes the linear subspace of $X$ spanned by $E$. For $x \in X$, we write:

$$
\|x-E\|=\inf \{\|x-y\|: y \in E\} .
$$

For undefined notation, see [Bo, BP, Re].

\section{THE LOCALLY CONVEX APPROXIMATION PROPERTY AND ADMISSIBLE CONVEX SETS}

Following Klee [K1, K2] a convex set $X$ is admissible iff for every compact subset $A$ of $X$ and for every $\varepsilon>0$ there exists a map $f$ from $A$ into a finite-dimensional subset of $X$ such that $\|x-f(x)\|<\varepsilon$ for every $x \in A$.

The notion of admissibility is quite useful for detecting the AR-property for compact convex sets in linear metric spaces. In fact, Klee [K1, K2] proved that every admissible convex set $X$ has the compact extension property, that is, any map into $X$ defined on a compact subset of a metric space extends to the whole space. In particular, any admissible compact convex set is an AR. Using this property, we have discovered the AR-property for certain Roberts spaces [R1, R2]; see [NT1]. (By a Roberts space we mean any compact convex set with no extreme points constructed by Roberts' method of needle point spaces; see [NT1, NT2, N2, NST] for some results about the AR-property and the fixed point property for Roberts spaces.) Unfortunately, as shown in [BM], admissibility does not bring any information about the AR-property for noncompact convex sets. Our feeling is that the notion of admissibility should improve in order that we could have a better range of applications, especially to the noncompact case. The notion of the LCAP introduced in this section comes from this context.

2.1. Definition. A convex set $X$ in a linear metric space is $L C$-convex iff for every $\varepsilon>0$ there exists a $\delta=\delta(\varepsilon, X)>0$ such that for every finite subset $A \subset X$ with $\operatorname{diam} A<\delta$ we have $\operatorname{diam} \operatorname{conv} A<\varepsilon$.

Clearly, any convex set in a locally convex linear metric space is LC-convex.

We have the following obvious observations:

2.2. Fact. If $E$ is a LC-convex subset of a linear metric space $X$, then the closure $\bar{E}$ of $E$ in $X$ is also LC-convex.

From the Dugundji theorem (see [D, BP]), we get 
2.3. Fact. If $E$ is an LC-convex closed subset of a linear metric space $X$, then there is a Dugundji retraction $r: X \rightarrow E$ such that

$$
\|r(x)-x\|<3 \varepsilon \text { whenever } x \in X \text { with }\|x-E\|<\delta=\delta(\varepsilon, E) .
$$

Now we come to our main definition.

2.4. Definition. Let $X$ be a convex set lying in a linear metric space $Y$. We say that $X$ has the locally convex approximation property (the LCAP) iff there exist an $F$-norm \|\| on $Y$, a sequence $\left\{X_{n}\right\}$ of LC-convex subsets of $X$, and a sequence of maps $r_{n}: X \rightarrow X_{n}$ such that for some summable sequence $\left\{a_{n}\right\}$ of positive numbers we have

$$
\lim _{n \rightarrow \infty} \inf \left(a_{n}\right)^{-1}\left\|x-r_{n}(x)\right\|=0 \text { for every } x \in X .
$$

Of course every convex set in a locally convex space has the LCAP.

Our result in this section shows that the LCAP is in fact an extension of admissibility of Klee. Namely, we have the following theorem.

2.5. Theorem. Any convex set with the LCAP is admissible. Conversely, any admissible compact convex set has the LCAP.

Proof. Assume that $X$ is a convex set with the LCAP. Let $A$ be a compact subset of $X$, and let $\varepsilon>0$. Using the notation of Definition 2.4, we take, for every $x \in A$, a neighbourhood $U(x)$ of $x$ in $A$ and $n(x) \in \mathbb{N}$ such that

$$
\left(a_{n(x)}\right)^{-1}\left\|y-r_{n(x)}(y)\right\|<(2 a)^{-1} \varepsilon \text { for every } y \in U(x),
$$

where $a=\sum_{n=1}^{\infty} a_{n}$.

Since $A$ is compact, there exist finite points $x_{i} \in A, i=1, \ldots, n$, such that $A=\bigcup_{i=1}^{n} U\left(x_{i}\right)$. Denote

$$
\begin{gathered}
\{n(1), \ldots, n(k)\}=\left\{n\left(x_{i}\right): i=1, \ldots, n\right\}, \quad \text { where } n(1)<\cdots<n(k) ; \\
U_{i}=\bigcup\left\{U\left(x_{j}\right), n\left(x_{j}\right)=n(i)\right\}, \quad i=1, \ldots, k ; \\
f_{i}=r_{n(i)}: X \rightarrow X_{n(i)}, \quad i=1, \ldots, k .
\end{gathered}
$$

Then from (3) we get

(4) $\left\|x-f_{i}(x)\right\|<a_{n(i)}(2 a)^{-1} \varepsilon$ for every $x \in U_{i}$ and $i=1, \ldots, k$.

Observe that $\mathscr{U}=\left\{U_{1}, \ldots, U_{k}\right\}$ is a finite open cover of $A$. Let $\left\{\lambda_{1}, \ldots, \lambda_{k}\right\}$ be a partition of unity inscribed into $\mathscr{U}$.

Since $X_{n(i)}$ is LC-convex, there exists a $\delta_{i}>0$ such that for every finite set $F \subset X_{n(i)}$ with $\operatorname{diam} F<\delta_{i}$ we have diam conv $F<a_{n(i)}(2 a)^{-1} \varepsilon$.

Let $\mathscr{U}_{i}=\left\{U_{i j}, j=1, \ldots, k(i)\right\}$ be a finite open cover of $f_{i}(A)$ such that

$$
\operatorname{diam} U<2^{-1} \delta_{i} \text { for every } U \in \mathscr{U}_{i} \text {. }
$$

Let $\left\{\lambda_{i j}: j=1, \ldots, k(i)\right\}$ be a partition of unity inscribed into $\mathscr{U}_{i}$. For every $j=1, \ldots, k(i), i=1, \ldots, k$, select $x_{i j} \in U_{i j}$ and denote

$$
D=\operatorname{conv}\left\{x_{i j}, j=1, \ldots, k(i), i=1, \ldots, k\right\} \subset X,
$$

We define a map $\varphi_{i}: f_{i}(A) \rightarrow D$ by the formula

$$
\varphi_{i}(x)=\sum_{j=1}^{k(i)} \lambda_{i j}(x) x_{i j} \quad \text { for every } x \in f_{i}(A) .
$$


Then we have

$$
\left\|\varphi_{i}(x)-x\right\|<a_{n(i)}(2 a)^{-1} \varepsilon \quad \text { for every } x \in f_{i}(A) .
$$

Observe that $D$ is a finite-dimensional compact convex set of $X$. We define a map $r: A \rightarrow D$ by the formula

$$
r(x)=\sum_{i=1}^{k} \lambda_{i}(x) \varphi_{i} f_{i}(x) \quad \text { for every } x \in A .
$$

Then from (4), (5) we get

$$
\begin{aligned}
\|r(x)-x\| & =\left\|\sum_{i=1}^{k} \lambda_{i}(x) \varphi_{i} f_{i}(x)-x\right\| \\
& \leq\left\|\sum_{i \in I(x)} \lambda_{i}(x)\left(\varphi_{i} f_{i}(x)-x\right)\right\| \quad\left(\text { where } I(x)=\left\{i: x \in U_{i}\right\}\right) \\
& \leq \sum_{i \in I(x)}\left(\left\|\varphi_{i} f_{i}(x)-f_{i}(x)\right\|+\left\|f_{i}(x)-x\right\|\right) \\
& <2 \sum_{i \in I(x)} a_{n(i)}(2 a)^{-1} \varepsilon<2 \sum_{n=1}^{\infty} a_{n}(2 a)^{-1} \varepsilon=\varepsilon .
\end{aligned}
$$

Consequently, $X$ is admissible.

Conversely, assume that $X$ is an admissible compact convex set. Then for every $n \in \mathbb{N}$ there exists a map $f_{n}$ from $X$ into a finite-dimensional convex subset $X_{n}$ of $X$ such that

$$
\left\|f_{n}(x)-x\right\|<2^{-n} \text { for every } x \in X .
$$

Since $\left\{a_{n}\right\}=\left\{n 2^{-n}\right\}$ is a summable sequence, we infer that $X$ has the LCAP. The theorem is proved.

2.6. Remark. In the next section we shall prove that any convex set with the LCAP is an AR. Therefore, from [BM] it follows that the compactness assumption in the converse part of Theorem 2.5 is essential unless all linear metric spaces are AR. In other words if there exists a non-AR linear metric space then there exists an admissible convex set which does not have the LCAP.

\section{THE AR-PROPERTY FOR CONVEX SETS WITH THE LCAP}

In this section we prove that the LCAP does imply the AR-property for all convex sets (not necessarily separable) in linear metric spaces. Since every convex set in a locally convex space has the LCAP, our result is an extension of Dugundji theorem.

\subsection{Theorem. Any convex set with the LCAP is an AR.}

The proof of Theorem 3.1 is based on the following characterization of ANRspaces (see [N1]).

Let $X$ be a metric space. For an open cover $\mathscr{U}$ of $X$ let $\mathscr{N}(\mathscr{U})$ denote the nerve of $\mathscr{U}$ equipped with the Whitehead topology. Let $\left\{\mathscr{U}_{n}\right\}$ be a sequence of open covers of $X$. We say that $\left\{\mathscr{U}_{n}\right\}$ is a zero sequence iff

$$
\sup \left\{\operatorname{diam} U: U \in \mathscr{U}_{n}\right\} \rightarrow 0 \quad \text { as } n \rightarrow \infty \text {. }
$$


We denote

$$
\mathscr{U}=\bigcup_{n=1}^{\infty} \mathscr{U}_{n} \text { and } \mathscr{K}(\mathscr{U})=\bigcup_{n=1}^{\infty} \mathscr{N}\left(\mathscr{U}_{n} \cup \mathscr{U}_{n+1}\right) .
$$

For every $\sigma \in \mathscr{K}(\mathscr{U})$ we write

$$
n(\sigma)=\sup \left\{n \in \mathbb{N}: \sigma \in \mathcal{N}\left(\mathscr{U}_{n} \cup \mathscr{U}_{n+1}\right)\right\}
$$

We say that a map $f: \mathscr{U} \rightarrow X$ is a selection iff $f(U) \in U$ for every $U \in \mathscr{U}$.

The characterization of ANRs established by the author in [N1] is simplified to the following due to observations of J. Luukkainen and K. Sakai:

3.2. Theorem ([N1]; see also [NSk]). A metric space $X$ with no isolated points is an $A N R$ if and only if there exists a zero sequence of open covers $\left\{\mathscr{U}_{n}\right\}$ of $X$ such that for any selection $g: \mathscr{U} \rightarrow X$ there is a map $f: \mathscr{K}(\mathscr{U}) \rightarrow X$ such that for any sequence $\left\{\sigma_{k}\right\}$ of simplices of $\mathscr{K}(\mathscr{U})$ for which $n\left(\sigma_{k}\right) \rightarrow \infty$ we have $\operatorname{diam}\left(f\left(\sigma_{k}\right) \cup g\left(\sigma_{k}^{0}\right)\right) \rightarrow 0$ where $\sigma^{0}$ denotes the set of all vertices of $\sigma$.

We need the following simple fact.

3.3. Lemma. Let $X$ be a convex set in a linear metric space, and let $r: X \rightarrow E$ be a map from $X$ into an $L C$-convex subset $E$ of $X$. Then for every $\varepsilon>0$ there exists a family $\mathscr{U}$ of open subsets of $X$ with the following properties:

(i) $\operatorname{diam} U<\varepsilon$ for every $U \in \mathscr{U}$;

(ii) $\operatorname{diam} r(U)<3^{-1} \delta$ for every $U \in \mathscr{U}$, where $\delta=\delta(\varepsilon, E)>0$ (see 2.1);

(iii) $\|x-r(x)\|<3^{-1} \varepsilon$ if and only if $x \in U$ for some $U \in \mathscr{U}$.

Proof. Let $\mathscr{V}$ be an open cover of $E$ such that

$$
\operatorname{diam} V<3^{-1} \delta \text { for every } V \in \mathscr{V} \text {. }
$$

For every $V \in \mathscr{V}$ we denote

$V_{\varepsilon}=\left\{x \in r^{-1}(V):\|x-r(x)\|<3^{-1} \varepsilon\right\}, \quad \mathscr{U}=\left\{U: U=V_{\varepsilon}\right.$ for some $\left.V \in \mathscr{V}\right\}$.

Since $\delta=\delta(\varepsilon, E) \leq \varepsilon$, it is easy to see that $\mathscr{U}$ satisfies conditions (i)-(iii), and the lemma is proved.

Now we are already in a position to prove Theorem 3.1 .

Assume that $X$ is a convex set with the LCAP. Let $\left\{X_{n}\right\}$ be a sequence of LC-convex subsets of $X$, let $r_{n}: X \rightarrow X_{n}$ be a sequence of maps, and $\left\{a_{n}\right\}$ be a summable sequence of positive numbers satisfying condition (LC).

Applying Lemma 3.3 to $\varepsilon_{n}=a_{n}$ and $\delta_{n}=\delta\left(\varepsilon_{n}, X_{n}\right)$ we get a sequence $\left\{\mathscr{V}_{n}\right\}$ of families of open subsets of $X$ with the following properties:

$$
\begin{gathered}
\operatorname{diam} V<\varepsilon_{n}=a_{n} \quad \text { for every } V \in \mathscr{V}_{n} ; \\
\operatorname{diam} r_{n}(V)<3^{-1} \delta_{n} \quad \text { for every } V \in \mathscr{V}_{n} ; \\
\left\|x-r_{n}(x)\right\|<3^{-1} \varepsilon_{n} \quad \text { if and only if } \quad x \in V \text { for some } V \in \mathscr{V}_{n} .
\end{gathered}
$$

For every $n \in \mathbb{N}$ we denote $\widehat{\mathscr{V}_{n}}=\bigcup_{i=n}^{\infty} \mathscr{V}_{i}$ and $\widehat{\mathscr{V}}=\widehat{\mathscr{V}_{1}}$.

We claim that $\left\{\widehat{\mathscr{V}_{n}}\right\}$ is a zero sequence of open covers of $X$. In fact, let $n \in \mathbb{N}$ and $x \in X$. By (LC) there exists a sequence $\left\{n_{k}\right\} \subset \mathbb{N}$ such that

$$
\left\|x-r_{n_{k}}(x)\right\|<3^{-1} a_{n_{k}}=3^{-1} \varepsilon_{n_{k}} \quad \text { for every } k \in \mathbb{N} \text {. }
$$


Choose $k \in \mathbb{N}$ so that $n_{k} \geq n$. By (13) there exists $V \in \mathscr{V}_{n_{k}}$ such that $x \in V$. Whence, $V \in \widehat{\mathscr{V}}_{n}$, and from (11) we have

$$
\sup \{\operatorname{diam} V: V \in \widehat{\mathscr{V}}\} \leq \sup \left\{a_{i}: i \geq n\right\} \rightarrow 0 \text { as } n \rightarrow \infty .
$$

Therefore $\left\{\widehat{\mathscr{V}_{n}}\right\}$ is a zero sequence and the claim is proved.

We shall check that the sequence $\left\{\widehat{\mathscr{V}_{n}}\right\}$ satisfies the conditions of Theorem 3.2. Observe that $\left\{\widehat{\mathscr{V}_{n}}\right\}$ is a decreasing sequence. Therefore

$$
\widehat{\mathscr{V}}=\bigcup_{n=1}^{\infty} \widehat{\mathscr{V}}=\widehat{\mathscr{V}} \widehat{\mathbb{V}}_{1} \quad \text { and } \quad \mathscr{K}(\widehat{\mathscr{V}})=\mathscr{N}(\widehat{\mathscr{V}}) \quad(\text { see }(10)) \text {. }
$$

And for each $\sigma \in \mathscr{K}(\widehat{\mathscr{V}})$, we have

$$
n(\sigma)=\sup \{n \in \mathbb{N}: \sigma \in \mathscr{N}(\widehat{\mathscr{V}})\} .
$$

Let $g: \widehat{\mathscr{V}} \rightarrow X$ be a selection. First we define a map $f: \widehat{\mathscr{V}} \rightarrow X$ as follows: For every $V \in \widehat{\mathscr{V}}$, we have $V \in \mathscr{V}_{n}$ for some $n \in \mathbb{N}$. We let $f(V)=r_{n}(g(V)) \in$ $X_{n}$. Since $g(V) \in V$, from (13) we get

$$
\|f(V)-g(V)\|=\left\|r_{n}(g(V))-g(V)\right\|<3^{-1} \varepsilon_{n}=3^{-1} a_{n} .
$$

Then we extend $f$ by the convexity to the map, which is still denoted by $f$, $f: \mathscr{K}(\widehat{\mathscr{V}}) \rightarrow X$. We claim that $f$ satisfies the condition of Theorem 3.2.

Observe that $f(\mathscr{K}(\widehat{\mathscr{V}})) \subset \bigcup_{n=1}^{\infty} \operatorname{conv}\left(X_{n} \cup X_{n+1}\right)$.

First we compute diam $f(\sigma)$. For every $\sigma=\left\langle V_{1}, \ldots, V_{k}\right\rangle \in \mathscr{K}(\widehat{\mathscr{V}})$, take a finite sequence $\left\{m_{i}\right\}_{i=0}^{p} \subset \mathbb{N}$, with $m_{0}=0 \leq m_{1} \leq \cdots \leq m_{p}=k$, such that

$$
V_{m_{i}+1}, \ldots, V_{m_{i+1}} \in \mathscr{V}_{n(\sigma)+i} \text { for every } i=0,1, \ldots, p-1 \text {, }
$$

and $f\left(V_{j}\right)=r_{n(\sigma)+i}\left(g\left(V_{j}\right)\right) \in X_{n(\sigma)+i}$ if $m_{i}<j \leq m_{i+1}$.

Observe that for every $x \in \operatorname{conv}\left\{f\left(V_{j}\right): j=1, \ldots, k\right\}$, we have

$$
x=\sum_{j=1}^{k} \lambda_{j} f\left(V_{j}\right)=\sum_{i=0}^{p-1} \sum_{j=m_{i}+1}^{m_{i+1}} \lambda_{j} f\left(V_{j}\right),
$$

where $\lambda_{j} \geq 0, j=1, \ldots, k$, and $\sum_{j=1}^{k} \lambda_{j}=1$.

Denote

$$
\alpha_{i}=\sum_{j=m_{i}+1}^{m_{i+1}} \lambda_{j} \text { for } i=0, \ldots, p-1,
$$

and

$$
\mu_{i j}= \begin{cases}\left(\alpha_{i}\right)^{-1} \lambda_{j} & \text { if } \alpha_{i}>0 \\ 0 & \text { if } \alpha_{i}=0\end{cases}
$$

Then we have

$$
x=\sum_{i=0}^{p-1} \alpha_{i} \sum_{j=m_{i}+1}^{m_{i+1}} \mu_{i j} f\left(V_{j}\right),
$$

where $\sum_{i=0}^{p-1} \alpha_{i}=1$ and $\sum_{j=m_{i}+1}^{m_{i+1}} \mu_{i j}=1$ for $i=0, \ldots, p-1$.

Note that $\bigcap_{j=1}^{k} V_{j} \neq \varnothing$. For every $i=0, \ldots, p-1$, select $b_{i} \in \bigcap_{j=m_{i}+1}^{m_{i+1}} V_{j}$ and denote $a_{i}=r_{n(\sigma)+i}\left(b_{i}\right) \in X_{n(\sigma)+i}$. 
Take $a \in \bigcap_{j=1}^{k} V_{j}$. Observe that

$$
\begin{aligned}
x-a= & \sum_{i=0}^{p-1} \alpha_{i} \sum_{j=m_{i}+1}^{m_{i+1}} \mu_{i j}\left(f\left(V_{j}\right)-a_{i}\right) \\
& +\sum_{i=0}^{p-1} \alpha_{i} \sum_{j=m_{i}+1}^{m_{i+1}} \mu_{i j}\left(a_{i}-a\right) \\
= & \sum_{i=0}^{p-1} \alpha_{i} \sum_{j=m_{i}+1}^{m_{i+1}} \mu_{i j}\left(f\left(V_{j}\right)-a_{i}\right)+\sum_{i=0}^{p-1} \alpha_{i}\left(a_{i}-a\right) .
\end{aligned}
$$

Since $a_{i}, f\left(V_{j}\right) \in r_{n(\sigma)+i}\left(V_{j}\right)$ for $j=m_{i}+1, \ldots, m_{i+1}$, from (12) we get $\operatorname{diam}\left\{a_{i}, f\left(V_{j}\right), j=m_{i}+1, \ldots, m_{i+1}\right\}<\delta_{n(\sigma)+i}$ for every $i=0,1, \ldots, p-1$. Since $\delta_{n(\sigma)+i}=\delta\left(\varepsilon_{n(\sigma)+i}, X_{n(\sigma)+i}\right)$ (see 2.1), we have diam conv $\left\{a_{i}, f\left(V_{j}\right): j=\right.$ $\left.m_{i}+1, \ldots, m_{i+1}\right\}<\varepsilon_{n(\sigma)+i}$ for every $i=0,1, \ldots, p-1$. From (11) and (13) we get

$$
\begin{aligned}
\left\|a-a_{i}\right\| & =\left\|a-r_{n(\sigma)+i}\left(b_{i}\right)\right\| \\
& \leq\left\|a-b_{i}\right\|+\left\|b_{i}-r_{n(\sigma)+i}\left(b_{i}\right)\right\| \\
& <\varepsilon_{n(\sigma)+i}+3^{-1} \varepsilon_{n(\sigma)+i}<2 \varepsilon_{n(\sigma)+i} .
\end{aligned}
$$

Hence from (16) we obtain

$$
\begin{aligned}
\operatorname{diam} f(\sigma) & =\operatorname{diam} \operatorname{conv}\left\{f\left(V_{j}\right): j=1, \ldots, k\right\} \\
& \leq 2 \sup \left\{\|x-a\|: x \in \operatorname{conv}\left\{f\left(V_{j}\right): j=1, \ldots, k\right\}\right\} \\
& \leq 2 \sum_{i=0}^{p-1}\left(\left\|a_{i}-a\right\|+\operatorname{diam} \operatorname{conv}\left\{a_{i}, f\left(V_{j}\right): j=m_{i}+1, \ldots, m_{i+1}\right\}\right) \\
& \leq 2 \sum_{i=0}^{p-1}\left(2 \varepsilon_{n(\sigma)+i}+\varepsilon_{n(\sigma)+i}\right)=6 \sum_{i=0}^{p-1} \varepsilon_{n(\sigma)+i} \\
& <6 \sum_{n=n(\sigma)}^{\infty} \varepsilon_{n}=6 \sum_{n=n(\sigma)}^{\infty} a_{n} .
\end{aligned}
$$

From (11) we get also

$$
\begin{aligned}
\operatorname{diam} g\left(\sigma^{0}\right) & \leq 2 \max \left\{\left\|g\left(V_{j}\right)-a\right\|: j=1, \ldots, k\right\} \\
& \leq 2 \max \left\{\operatorname{diam} V_{j}: j=1, \ldots, k\right\} \\
& <2 \sum_{n=n(\sigma)}^{\infty} a_{n} .
\end{aligned}
$$

Consequently, from (14) we get

$$
\begin{aligned}
\operatorname{diam}\left(f(\sigma) \cup g\left(\sigma^{0}\right)\right) & \leq \operatorname{diam} f(\sigma)+3^{-1} a_{n(\sigma)}+\operatorname{diam} g\left(\sigma^{0}\right) \\
& <9 \sum_{n=n(\sigma)}^{\infty} a_{n} .
\end{aligned}
$$

Since $\left\{a_{n}\right\}$ is a summable sequence, we have $\operatorname{diam}\left(f(\sigma) \cup g\left(\sigma^{0}\right)\right) \rightarrow 0$ as $n(\sigma) \rightarrow \infty$. Consequently, we have $X \in A N R$ by Theorem 3.2. Since $X$ is contractible, it follows that $X \in A R$, and the proof of Theorem 3.1 is finished. 
It is of interest to know that the LCAP implies the AR-property for all convex sets even in the nonseparable situation, meanwhile admissibility does not bring any information about the AR-property for noncompact convex sets. So the LCAP is really a "good" extension of admissibility.

From Theorem 3.1 and from Facts 2.2 and 2.3 we obtain

\subsection{Corollary. Any $\sigma$-LC-convex set is an AR.}

Here we say that a convex set $X$ is $\sigma$-LC-convex iff $X$ is a union of an increasing sequence of LC-convex subsets of $X$.

Proof. Assume that $X=\bigcup_{n=1}^{\infty} X_{n}$, where $\left\{X_{n}\right\}$ is an increasing sequence of LC-convex subsets of $X$. By 2.2 we may assume that $X_{n}$ is closed in $X$ for every $n \in \mathbb{N}$. By 2.3 for every $n \in \mathbb{N}$ there exists a Dugundji retraction $r_{n}: X \rightarrow X_{n}$. Since $\left\{X_{n}\right\}$ is an increasing sequence, for every $x \in X$ there exists an $n(x) \in \mathbb{N}$ such that $r_{n}(x)=x$ for every $n \geq n(x)$. Consequently, $X$ has the LCAP, therefore is an AR by Theorem 3.1. The corollary is proved.

Obviously every separable convex set contains a dense $\sigma$-LC convex subset. Therefore, from Corollary 3.4 it follows that an affirmative answer to the following question would imply the AR-property for all separable convex sets.

3.5. Question. Let $\bar{X}$ denote the closure of a convex set $X$ lying in a linear metric space. Assume that $X$ has the LCAP. Has $\bar{X}$ the LCAP?

Observe that Theorem 3.1 reduces Problem 1.1 to

3.6. Question. Has every convex set the LCAP? What about separable convex sets? Compact convex sets?

In [N2] we introduced the notion of the finite-dimensional approximation property (the FDAP) for convex sets in linear metric spaces and proved that if $X$ is a convex set with the FDAP, then every convex subset of $X$ is an AR. We do not know whether the LCAP has this stronger property.

3.7. Question. Assume that $X$ is a convex set with the LCAP. Is every convex subset of $X$ an AR?

\section{ACKNOWLEDGMENT}

This paper was written while I was visiting the Complutense University of Madrid, 1990-1991. I would like to express my gratitude to the Spanish Ministry of Education for finanical support and thank the members of the Department of Geometry and Topology for their hospitality. Especially, I am very grateful to Jose M. R. Sanjurjo for many valuable discussions during the preparation of this paper. Thanks are due to Nguyen Nhuy and Tran Van An for their comments. Finally, I am very grateful to the referee for his suggestions for revising the paper.

\section{REFERENCES}

[BP] C. Bessaga and A. Pelczynski, Selected topics in infinite dimensional topology, Warszawa, 1975.

[BM] J. van der Bijl and J. van Mill, Linear spaces, absolute retracts and the compact extension property, Proc. Amer. Math. Soc. 104 (1988), 942-952. 
[Bo] K. Borsuk, Theory of retracts, Warszawa, 1967.

[D] J. Dugundji, An extension of Tietze's theorem, Pacific J. Math. 1 (1951), 353-367.

[G] R. Geoghegan, Open problems in infinite dimensional topology, Topology Proc. 4 (1979), 287-333.

[K1] V. Klee, Shrinkable neighbourhoods in Hausdorff linear spaces, Math. Ann. 141 (1960), 281-285.

[K2] Leray-Schauder theory without local convexity, Math. Ann. 141 (1960), 286-296.

[KM] M. G. Krein and D. P. Milman, On extreme points in regular convex sets, Studia Math. 9 (1940), 133-138.

[N1] Nguyen To Nhu, Investigating the ANR-property of metric spaces, Fund. Math. 124 (1984), 243-254; corrections, Fund. Math. 141 (1992), 297.

[N2] $\_$, The finite dimensional approximation property and the AR-property in needle point spaces, J. London Math.Soc. (to appear).

[NSk] Nguyen To Nhu and Katsuro Sakai, The compact neighbourhood extension property and the local equi-connectedness, Proc. Amer. Math. Soc. 121 (1994), 259-265.

[NST] Nguyen To Nhu, Jose M. R. Sanjurjo, and Tran Van An, The AR-property for Roberts' example of a compact convex set with no extreme points, Proc. Amer. Math. Soc. (submitted).

[NT1] Nguyen To Nhu and Le Hoang Tri, Every needle point space contains a compact convex AR-set with no extreme points, Proc. Amer. Math. Soc. 120 (1994), 1261-1265.

[NT2] _ No Roberts space is a counter-example to Schauder's conjecture, Topology 33 (1994), 371-378.

[R1] J. W. Roberts, A compact convex set with no extreme points, Studia Math. 60 (1977), 255-266.

[R2] Pathological compact convex sets in the spaces $L_{p}, 0 \leq p<1$, The Altgeld Book, University of Illinois, 1976.

[Re] S. Rolewicz, Metric linear spaces, Warszawa, 1972.

[W] J. E. West, Open problems in infinite dimensional topology, Open Problems in Topology (J. van Mill and G. M. Reed, eds.), Elsevier Science Publishers, B.V. (North-Holland), New York, 1990, pp. 523-597.

Institute of Mathematics at Hanoi, P.O. Box 631, Bo Ho, Hanol, Vietnam

Departamento de Geometria y Topologia, facultad de Matematicas, Universidad COMPLUTENSE, 28040 MADRID, SPAin

Current address: Department of Mathematics, Louisiana State University-Shreveport, One University Place, Shreveport, Louisiana 71115 